\title{
A lattice study of the nucleon quark content at the physical point
}

\author{
C. Torrero $^{* a}$, S. Durr ${ }^{b, c}$, Z. Fodor ${ }^{b, c}$, C. Hoelbling ${ }^{b}$, S. D. Katz ${ }^{d, e}$, S. Krieg ${ }^{b, c}$, \\ L. Lellouch ${ }^{a}$, T. Lippert ${ }^{b, c}$, T. Metivet ${ }^{a, f}$, A. Portelli ${ }^{a, g}$, K. K. Szabo ${ }^{b, c}$, B. C. Toth $^{b}$, \\ L. Varnhorst ${ }^{b}$
}

${ }^{a}$ CNRS, Aix-Marseille Université, Université de Toulon, Centre de Physique Théorique (CPT, UMR 7332), F-13288 Marseille, France

${ }^{b}$ Department of Physics, University of Wuppertal, D-42119 Wuppertal, Germany

${ }^{c}$ Jülich Supercomputing Centre, Forschungszentrum Jülich, D-52428 Jülich, Germany

${ }^{d}$ Institute for Theoretical Physics, Eötvös University, H-1117 Budapest, Hungary

e MTA-ELTE Lendület Lattice Gauge Theory Research Group, Budapest, Hungary

${ }^{f}$ CEA-Saclay, IRFU/SPhN, 91191 Gif-sur-Yvette France

${ }^{g}$ Higgs Centre for Theoretical Physics, School of Physics and Astronomy,

The University of Edinburgh, Edinburgh EH9 3FD, UK

E-mail: Christian.Torreroecpt.univ-mrs.fr

\begin{abstract}
After a detailed analysis of possible sources of systematic uncertainty, ab-initio $N_{f}=2+1$ results for the up-down and strange quark content - with pion masses all the way down to the physical point - are presented and discussed.
\end{abstract}

The 33rd International Symposium on Lattice Field Theory

14 -18 July 2015

Kobe International Conference Center, Kobe, Japan

\footnotetext{
* Speaker.
} 


\section{Introduction}

Due to their connection to the hadron spectrum, pion-nucleon and kaon-nucleon scattering, counting rates in Higgs boson searches and direct detection of Dark Matter, the nucleon $(N)$ updown and strange quark contents $f_{u d}^{N}$ and $f_{s}^{N}$ are important quantities from the phenomenological point of view. They are defined as

$$
\begin{aligned}
f_{u d}^{N} & =m_{u d} \frac{\langle N|\bar{u} u+\bar{d} d| N\rangle}{2 M_{N}^{2}} \equiv \frac{\sigma_{\pi N}}{M_{N}}, \\
f_{q}^{N} & =m_{q} \frac{\langle N|\bar{q} q| N\rangle}{2 M_{N}^{2}} \equiv \frac{\sigma_{q N}}{M_{N}}
\end{aligned}
$$

where $q=u, d, s$ and $m_{u d}=\left(m_{u}+m_{d}\right) / 2$. Recalling that they cannot be obtained directly from experiment, their first determinations come from phenomenology [1, 2]. However, they are affected by large uncertainties and agree only marginally within errorbars. This situation prompted new phenomenological studies [3]-[8] as well as a series of lattice computations [11]-[20] (recent reviews on $\sigma_{\pi N}$ can be found in [9] and [10]). However, it is still challenging to carry out a lattice computation which avoids any model assumption and includes a complete error analysis. By exploiting the Feynman-Hellmann theorem, this work displays an $a b$-initio study of the nucleon quark contents where the different sources of systematic uncertainty are carefully taken into account.

\section{Methodology}

The dataset at the basis of this study consists of 47 ensembles obtained through $N_{f}=2+1$ simulations performed with a tree-level-improved Symanzik gauge action and clover-improved Wilson quarks, the latter featuring 2 levels of HEX link smearing. The ensembles are made up of approximately 13000 configurations altogether, corresponding to 5 different lattice spacings $a$ (ranging from $0.054 \mathrm{fm}$ to $0.116 \mathrm{fm}$ ), lattice sizes up to $6 \mathrm{fm}$ and pion masses $M_{\pi}$ all the way down to $120 \mathrm{MeV}$. This setup allows for a consistent control of systematic uncertainties when reaching the physical point $(\Phi)$, i.e. when interpolating to physical $m_{u d}^{(\Phi)}$ and $m_{s}^{(\Phi)}$ and extrapolating to $a \rightarrow 0$ and $L \rightarrow \infty, L$ being the lattice spatial size.

Though a direct computation of the matrix elements in Eq.(1.1) could in principle benefit the precision on the results, such an approach entails taking into account disconnected quark contributions as well as an involved renormalization pattern. An alternative possibility is given by the Feynman-Hellmann theorem which connects $f_{u d}^{N}$ and $f_{s}^{N}$ to the derivatives of $M_{N}$ with respect to $m_{u d}$ and $m_{s}$ respectively. More precisely,

$$
f_{u d}^{N}=\left.\frac{m_{u d}}{M_{N}} \frac{\partial M_{N}}{\partial m_{u d}}\right|_{\Phi}, \quad f_{s}^{N}=\left.\frac{m_{s}}{M_{N}} \frac{\partial M_{N}}{\partial m_{s}}\right|_{\Phi} .
$$

This indirect approach is convenient since it only requires the computation of the nucleon and (renormalized) quark masses. The hadron masses of interest here can be extracted by fitting the asymptotic behaviour of suitable 2 -point time correlators $C(t)$ - which do not imply any disconnected diagrams - while quark masses can be obtained through the ratio-difference method 
described in [21]. Quark masses are then renormalized non-perturbatively in the Renormalization Group Invariance (RGI) scheme, using a generalization of the Rome-Southampton method as explained in [21]. Gaussian sources and sinks are employed in the evaluation of $C(t)$ while, in applying the ratio-difference method, sources are Gaussian while sinks are point-like.

\section{Data analysis}

In computing the derivatives in Eq. (2.1), it is important to notice that usually Chiral Perturbation Thoery $(\chi \mathrm{PT})$ is invoked and the dependence with respect to $m_{u d}$ and $m_{s}$ is traded for the dependence with respect to $M_{\pi}^{2}$ and $M_{K^{x}}^{2}=M_{K}^{2}-M_{\pi}^{2} / 2$. However, in the present study, the quality of the dataset at disposal allows for avoiding any $\chi$ PT-related assumption (which might entail some systematics, especially for $f_{s}^{N}$ ) and for studying the contributions of quark masses to hadron masses explicitly. Since $m_{u d}^{(\Phi)}$ and $m_{s}^{(\Phi)}$ cannot be measured experimentally, they need to be fixed by fitting the quark-mass dependence of two hadron masses - other than $M_{N}$ - whose physical value is known: a convenient choice is given by $M_{\pi}^{2}$ and $M_{K^{\chi}}$. Besides $M_{N}, M_{\pi}^{2}$ and $M_{K \chi}$, a fourth observable is needed in order to set the scale and, as often the case, the $\Omega$ baryon mass, $M_{\Omega}$, turns out to be appropriate.

In the fit of $C(t)$, assessing when the asymptotic behaviour sets in is a non-trivial task, usually carried out by relying on eye judgement and/or on the fit quality. However, the latter being a stochastic variable on its own, a more systematic approach is given by the Kolmogorov-Smirnov (KS) test [23] which consists in comparing the empirical distribution function $E(x)$ of a stochastic variable $x$ with a reference distribution $F(x)$. After determining their maximal distance $D$ defined as $D=\sup _{x}|F(x)-E(x)|$, a significance level $P$ is computed. In the present work, for a given hadron mass, the initial time $t_{\min }$ in physical units is fixed together with the length $\Delta t$ (in lattice units) of the time interval where the asymptotics of $C(t)$ is then fitted on the 47 ensembles. Afterwards, the KS test is applied to the distribution of the corresponding fit qualities and, if $P$ is larger than 0.3 , the combination $\left(t_{\min }, \Delta t\right)$ is considered good. Otherwise the test is repeated by varying either $t_{\min }$ or $\Delta t$ or both. This procedure is separately applied to all masses needed in the study.

Once that the mass of hadron $X$ in lattice units has been obtained, its dependence on $m_{u d}$ and $m_{s}$ has to be parametrized. This is carried out by means of a function like

$\left(a M_{X}\right)^{n_{X}}=a^{n_{X}}\left(1+g_{X}^{\mathrm{a}}(a)\right)\left(1+g_{X}^{\mathrm{FV}}\left(M_{\pi}, L\right)\right)\left(M_{X}^{(\Phi)}\right)^{n_{X}}\left[1+c_{X}^{a, u d}(a) \tilde{m}_{u d}+c_{X}^{a, s}(a) \tilde{m}_{s}+\right.$ h.o.t. $]$

where $X=N, \Omega, \pi, K^{\chi}$ with $n_{X}=2$ for $X=\pi$ and $n_{X}=1$ otherwise. Quark mass terms are given by $\tilde{m}_{q}=m_{q}^{R G I}-m_{q}^{(\Phi)}(q=u d, s)$ with $m_{q}^{R G I}=a m_{q} Z_{s}^{-1}(\beta) / a\left(1+g_{q}^{a}(a)\right), Z_{s}(\beta)$ being the non-perturbative renormalization constant in the RGI scheme computed in a dedicated study [21]. In Eq. (3.1), h.o.t. denotes higher-order terms in the Taylor expansion while coefficients $g_{X}^{\mathrm{a}}(a)$ and $g_{X}^{\mathrm{FV}}\left(M_{\pi}, L\right)$ parametrize discretization and finite-volume corrections respectively, the latter modelled in the spirit of $[24,25]$. Coefficients $c_{X}^{a, q}(a)(q=u d, s)$ are given by $c_{X}^{a, q}(a)=c_{X}^{q}\left(1+g_{X}^{\mathrm{a}, q}(a)\right)$, where $g_{X}^{\mathrm{a}, q}(a)$ parametrizes the continuum extrapolation of the slope parameter $c_{X}^{q}$.

The only experimental input in Eq.(3.1) corresponds to $M_{\Omega}^{(\Phi)}, M_{\pi}^{(\Phi)}$ and $M_{K \chi}^{(\Phi)}$ (set to $1672.45 \mathrm{MeV}$, 

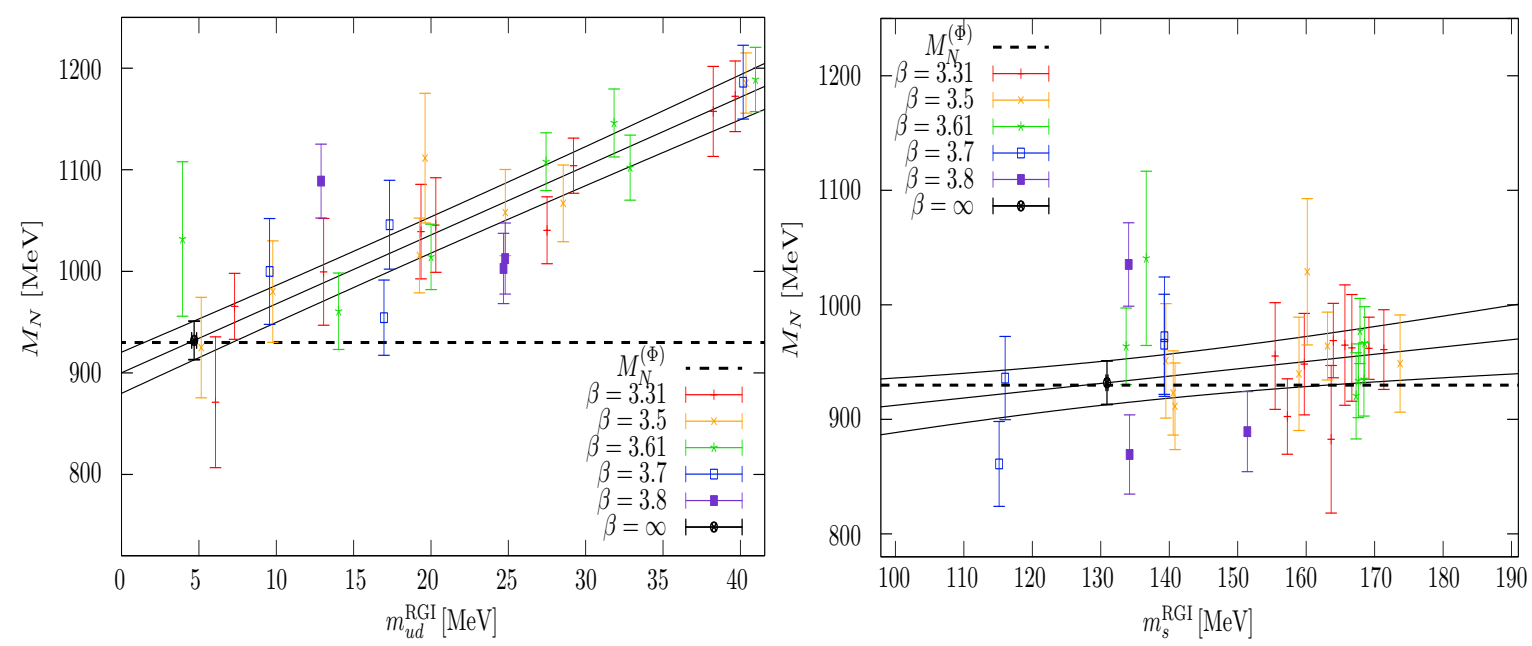

Figure 1: (Color online) Typical dependence of $M_{N}$ with respect to $m_{u d}^{R G I}$ (left) and $m_{s}^{R G I}$ (right). The black point represents the numerical result for $M_{N}^{(\Phi)}$ while the horizontal line corresponds to its experimental value.

134.8 MeV and 484.9 MeV[26] respectively ${ }^{1}$ ), all other coefficients being fit parameters to be determined by minimizing a standard $\chi^{2}$ function where errorbars on data have been obtained through a bootstrap procedure with 2000 samples. All four hadron masses are fitted at the same time: note that some coefficients in Eq.(3.1) - namely $m_{u d}^{(\Phi)}, m_{s}^{(\Phi)}, g_{q}^{a}(a)$ and the lattice spacings — are in common. All fits are correlated, including those of $C(t)$ described previously.

\section{Evaluation of statistical and systematic uncertainties}

In order to carry out an ab-initio study, it is necessary not to make any a priori assumptions and to assess the impact of the different sources of systematic error. This goal is accomplished by allowing for variations at different stages of the data analysis as in [27].

As for the fit of the 2-point time correlators, two different time ranges have been considered, the first corresponding to the setup $\left(t_{\min }, \Delta t\right)$ obtained through the KS test, the second obtained by shifting $t_{\min }$ forward by one timeslice for each particle mass while keeping $\Delta t$ fixed. Truncation errors in the Taylor expansion have been taken into account by pruning the data with two cuts in the pion mass at $320 \mathrm{MeV}$ and $480 \mathrm{MeV}$. In addition to this, higher-order terms proportional to $\tilde{m}_{u d}^{2}$ (or also a $\chi$ PT-inspired $\left[\left(m_{u d}^{\mathrm{RGI}}\right)^{3 / 2}-\left(m_{u d}^{(\Phi)}\right)^{3 / 2}\right]$ for $\left.M_{N}\right), \tilde{m}_{u d}^{3}, \tilde{m}_{u d} \tilde{m}_{s}$ and $\tilde{m}_{u d}^{2} \tilde{m}_{s}$ in (3.1) have been considered. Concerning cut-off effects, the improvement procedure should grant leading $O\left(\alpha_{s} a\right)$ corrections ( $\alpha_{s}$ being the strong-coupling constant) but, in practice, HEX smearing might result in $O\left(a^{2}\right)$ corrections being dominant: both possibilities are taken into account by setting $g_{N}^{\text {a }}(a)$ to be proportional to either $a^{2}$ or $\alpha_{s} a$. As for the uncertainties associated with the definition of $Z_{s}(\beta)$, this is accounted for by allowing for six different procedures to be alternatively employed in the renormalization process [21]. Finally, any further uncertainty is estimated by replacing the Taylor expansion in Eq.(3.1) with Padé approximants in the fit of $M_{N}$.

\footnotetext{
${ }^{1}$ Consequently, the corresponding discretization corrections are set to zero by definition, only $g_{N}^{\mathrm{a}}(a)$ is left.
} 

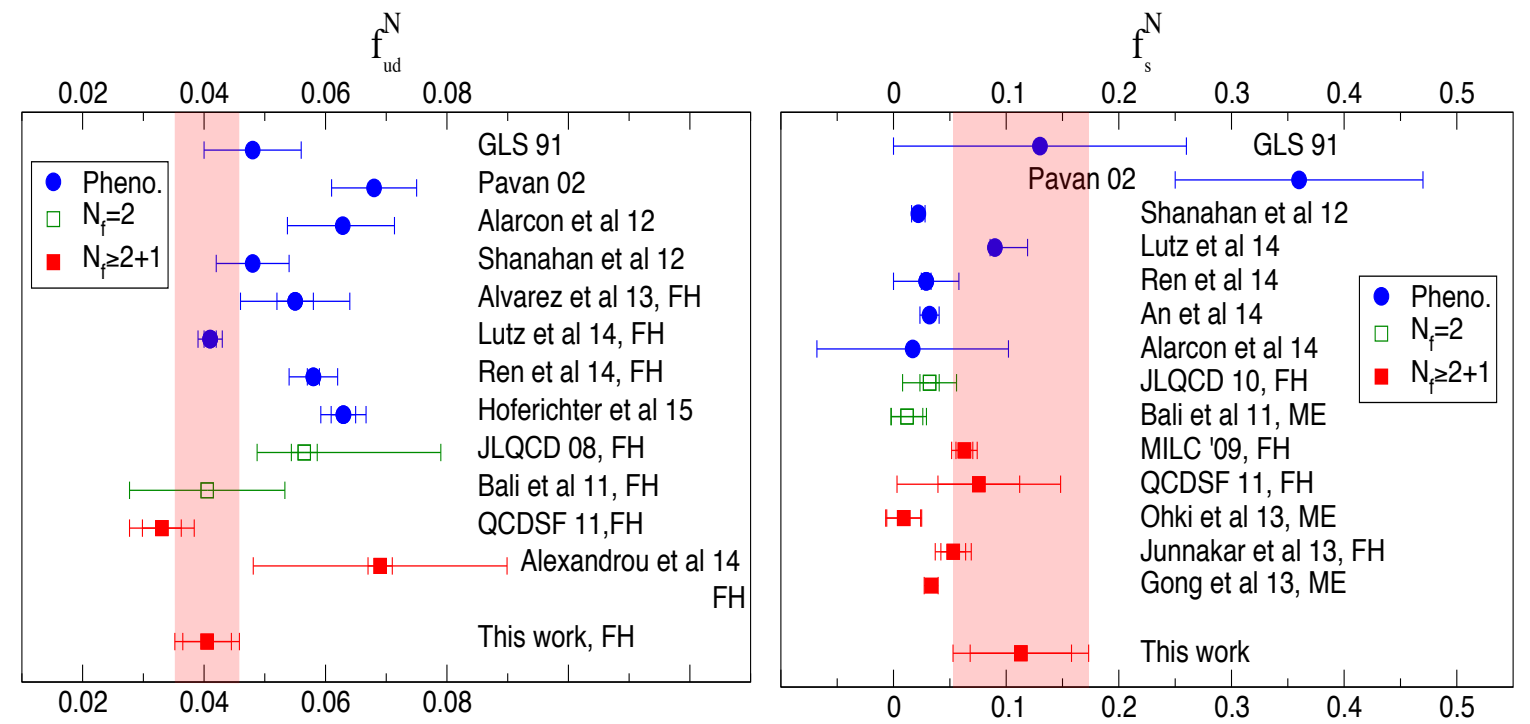

Figure 2: (Color online) Comparison of the results for $f_{u d}^{N}$ (left) and $f_{s}^{N}$ (right) obtained in this work with other studies.

This overall procedure leads to 192 different analyses, each one providing a result for $f_{u d}^{N}$ and $f_{s}^{N}$ as an output. These 192 values are subsequently weighed through Akaike's Information Criterion (AIC) [28], the AIC-weighed mean and standard deviation corresponding to the mean value and systematic error of the given observable respectively. The statistical error is computed through the bootstrap error on the AIC-weighed mean.

With such a procedure, the systematics associated with the continuum extrapolation of the leading $M_{N}^{(\Phi)}$ term in (3.1) is accounted for while the uncertainties related to the continuum extrapolation of the sub-leading $f_{u d}^{N}$ or $f_{s}^{N}$ contributions are not. Indeed, the discretization terms $g_{N}^{\mathrm{a}, q}(a)$, were considered to be zero in the above analyses. In order to take them into account, the terms $g_{N}^{\mathrm{a}, q}(a)$ were set to be proportional to either $\alpha_{s} a$ or $a^{2}$ and $M_{N}^{(\Phi)}$ was fixed to its experimental value to stabilize the corresponding fits. Even with such a setup, only corrections in $c_{N}^{\mathrm{a}, u d}(a)$ are supported by the data. By including these and by performing the same variation of 192 analyses as in the procedure described above, the central values of $f_{u d}^{N}$ increases by 0.0024 and $f_{s}^{N}$ decreases by 0.038 compared to the standard analysis. We use this variation to estimate the uncertainty associated with the continuum extrapolation of the quark contents. It has been added in quadrature to the systematic error obtained in the standard analysis and propagated throughout.

\section{Results and discussion}

A check of the accuracy of our approach is given by the comparison between the numerical result for $M_{N}^{(\Phi)}$ and its experimental value. Our estimate reads $M_{N}=929(16)(7) \mathrm{MeV}$ - the numbers between brackets are the statistical and systematic error in order - in good agreement with experiment $(938.9 \mathrm{MeV})$. An example of the typical dependence of $M_{N}$ with respect to $m_{u d}^{R G I}$ and $m_{s}^{R G I}$ is shown in Fig. (1). 
The final results for the quark contents are

$$
f_{u d}^{N}=0.0405(40)(35), \quad f_{s}^{N}=0.113(45)(40),
$$

which agree with most lattice computations within errorbars, as can be deduced from Fig. (2). Results obtained by weighing the analyses with a constant weight or with their fit quality are consistent with the values in Eq.(5.1).

While the precision on the light quark content is good (the combined error reads 13\%), the overall uncertainty on $f_{s}^{N}$ is still large, 53\% approximately. This is a consequence of the fact that, as is shown in the rightmost plot of Fig. (1), the dependence of $M_{N}$ with respect to $m_{s}$ is small, this being the major drawback of the present approach based on the Feynman-Hellmann theorem. In order to try to improve on the precision, the whole analysis has also been carried out by fixing $M_{N}^{(\Phi)}$ to its experimental value but the impact on the errorbars is not significant. To our understanding, the uncertainty on $f_{s}^{N}$ in the Feynman-Hellmann approach can be narrowed only by reducing the statistical error on the data and by increasing the lever arm on $m_{s}$.

\section{Acknowledgments}

Computations were performed using the JUGENE installation of FZ Jülich and HPC resources provided by GENCI-IDRIS (grant 52275), as well as further resources at FZ Jülich and clusters at Wuppertal and CPT. This work was supported in part by the OCEVU Labex (ANR-11-LABX0060), the A*MIDEX project (ANR-11-IDEX-0001-02) and by DFG grant SFB/TRR-55.

\section{References}

[1] J. Gasser, H. Leutwyler, and M. Sainio, Sigma-term update, Phys. Lett. B253 (1991) 252.

[2] M. Pavan, I. Strakovsky, R. Workman, and R. Arndt, The pion-nucleon Sigma term is definitely large: results from a G.W.U. analysis of pion nucleon scattering data, PiN Newslett. 16 (2002) 110, [hep-ph/0111066].

[3] J. M. Alarcon, J. Martin Camalich, and J. A. Oller, The chiral representation of the $\pi N$ scattering amplitude and the pion-nucleon sigma term, Phys.Rev. D85 (2012) 051503, [hep-ph/1110 . 3797].

[4] L. Alvarez-Ruso, T. Ledwig, J. Martin Camalich, and M. J. Vicente-Vacas, The nucleon mass and pion-nucleon sigma term from a chiral analysis of lattice QCD world data, Phys. Rev. D88 (2013) 054507 [hep-ph/1304.0483].

[5] X.-L. Ren, L.-S. Geng, and J. Meng, Scalar strangeness content of the nucleon and baryon sigma terms, Phys. Rev. D91 (2014) 051502 [hep-ph/1404 . 4799].

[6] M. Lutz, R. Bavontaweepanya, C. Kobdaj, and K. Schwarz, On finite volume effects in the chiral extrapolation of baryon masses, Phys. Rev. D90 (2014) 054505 [hep-lat/1401. 7805].

[7] J. M. Alarcon, L. S. Geng, J. Martin Camalich, and J. A. Oller, The strangeness content of the nucleon from effective field theory and phenomenology, Phys. Lett. B730 (2014) 342 [hep-ph/1209.2870].

[8] M. Hoferichter, J. Ruiz de Elvira, B. Kubis, and U.-G. Meissner, High-Precision Determination of the Pion-Nucleon $\sigma$ Term from Roy-Steiner Equations, Phys. Rev. Lett. 115 (2015) 9, 092301

[hep-ph/1506.04142]. 
[9] M. Hoferichter, J. Ruiz de Elvira, B. Kubis, and U.-G. Meissner, Roy-Steiner-equation analysis of pion-nucleon scattering, [hep-ph/1510.06039].

[10] H. Leutwyler, Theoretical aspects of Chiral Dynamics, [hep-ph/1510 . 07511].

[11] C. Alexandrou, et al. (ETM Collaboration), The low-lying baryon spectrum with two dynamical twisted mass fermions, Phys. Rev. D80 (2009) 114503 [hep-lat/ 0910.2419 ].

[12] D. Toussaint and W. Freeman (MILC), The strange quark condensate in the nucleon in $2+1$ flavor QCD, Phys. Rev. Lett. 103 (2009) 122002 [hep-lat/ 0905 . 2432].

[13] R. Babich, R. C. Brower, M. A. Clark, et al., Exploring strange nucleon form factors on the lattice, Phys. Rev. D85 (2012) 054510 [hep-lat/1012.0562].

[14] G. S. Bali, et al. (QCDSF Collaboration), The strange and light quark contributions to the nucleon mass from Lattice QCD, Phys. Rev. D85 (2012) 054502 [hep-lat/1111 . 1600].

[15] R. Horsley, et al. (QCDSF-UKQCD Collaborations), Hyperon sigma terms for $2+1$ quark flavours, Phys. Rev. D85 (2012) 034506 [hep-lat/1110 . 4971].

[16] S. Durr, Z. Fodor, T. Hemmert, C. Hoelbling, J. Frison, et al. (BMW Collaboration), Sigma term and strangeness content of octet baryons, Phys. Rev. D85 (2012) 014509 [hep-lat/1109.4265].

[17] W. Freeman et al. (MILC Collaboration), The intrinsic strangeness and charm of the nucleon using improved staggered fermions, Phys. Rev. D88 (2013) 054503 [hep-lat/1204 . 3866].

[18] P. Junnarkar, and A. Walker-Loud, The Scalar Strange Content of the Nucleon from Lattice QCD, Phys. Rev. D87 (2013) 114510 [hep-lat/1301.1114].

[19] M. Gong, et al. (XQCD), Strangeness and charmness content of nucleon from overlap fermions on 2+1-flavor domain-wall fermion configurations, Phys. Rev. D88 (2013) 014503 [hep-ph/1304.1194].

[20] C. Alexandrou, V. Drach, K. Jansen, C. Kallidonis, and G. Koutsou, Baryon spectrum with $N_{f}=2+1+1$ twisted mass fermions, Phys. Rev. D90(7) (2014) 074501 [hep-lat/1406.4310].

[21] S. Durr, Z. Fodor, C. Hoelbling, S. D. Katz, et al. (BMW Collaboration), Lattice QCD at the physical point: Simulation and analysis details, JHEP 1108 (2011) 148 [hep-lat/1011.2711].

[22] A. Crivellin, M. Hoferichter, M. Procura, Accurate evaluation of hadronic uncertainties in spin-independent WIMP-nucleon scattering: Disentangling two- and three-flavor effects, Phys. Rev. D89 (2014) 054021 [hep-ph/1312.4951].

[23] W. H. Press, S. A. Teukolsky, W. T. Vetterling, B. P. Flannery, Numerical Recipes in C++: The Art of Scientific Computing, Cambridge University Press, 2002.

[24] G. Colangelo, S. Durr, C. Haefeli, Finite volume effects for meson masses and decay constants, Nucl. Phys. B721 (2005) 136 [hep-lat/0503014].

[25] G. Colangelo, A. Fuhrer, C. Haefeli, The pion and proton mass in finite volume, Nucl. Phys. Proc. Suppl. 153 (2006) 41 [hep-lat/0512002].

[26] S. Aoki, Y. Aoki, C. Bernard, T. Blum, et al., Review of lattice results concerning low energy particle physics, Eur. Phys. J. C74 (2014) 2890 [hep-lat/1310.8555].

[27] S. Durr, Z. Fodor, J. Frison, C. Hoelbling, et al. (BMW Collaboration), Ab-initio Determination of Light Hadron Masses, Science 322 (2008) 1224 (2008) [hep-lat/ 0906.3599 ].

[28] Sz. Borsanyi, S. Durr, Z. Fodor, C. Hoelbling, et al. (BMW Collaboration), Ab initio calculation of the neutron-proton mass difference, Science 347 (2015) 1452 (2015) [hep-lat/1406.4088]. 